\title{
Arbitrary Divergence Speed of the Least-Squares Method in Infinite-Dimensional Inverse Ill-Posed Problems
}

R. D. Spies $^{1,2}$ and K. G. Temperini ${ }^{1,3}$

(1) Instituto de Matemática Aplicada del Litoral, IMAL, CONICET-UNL, Güemes 3450, S3000GLN, Santa Fe, Argentina.

(2) Departamento de Matemática, Facultad de Ingeniería Química, Universidad Nacional del Litoral, Santa Fe, Argentina.

(3) Departamento de Matemática, Facultad de Humanidades y Ciencias, Universidad Nacional del Litoral, Santa Fe, Argentina.

E-mail: rspies@santafe-conicet.gov.ar, ktemperini@santafe-conicet.gov.ar

\begin{abstract}
A standard engineering procedure for approximating the solutions of an infinite-dimensional inverse problem of the form $A x=y$, where $A$ is a given compact linear operator on a Hilbert space $X$ and $y$ is the given data, is to find a sequence $\left\{X_{N}\right\}$ of finite-dimensional approximating subspaces of $X$ whose union is dense in $X$ and to construct the sequence $\left\{x_{N}\right\}$ of least squares solutions of the problem in $X_{N}$. In [1], Seidman showed that if the problem is ill-posed, then, without any additional assumptions on the exact solution or on the sequence of approximating subspaces $X_{N}$, it cannot be guaranteed that the sequence $\left\{x_{N}\right\}$ will converge to the exact solution. In this article this result is extended in the following sense: it is shown that if $X$ is separable, then for any $y \in X, y \neq 0$ and for any arbitrarily given function $s: \mathbb{N} \rightarrow \mathbb{R}^{+}$ there exists an injective, compact linear operator $A$ and an increasing sequence of finitedimensional subspaces $X_{N} \subset X$ such that $\left\|x_{N}-A^{-1} y\right\| \geq s(N)$ for all $N \in \mathbb{N}$, where $x_{N}$ is the least squares solution of $A x=y$ in $X_{N}$.
\end{abstract}

Keywords: Least-squares method, Ill-posed inverse problem, nonconvergence. 


\section{Introduction}

A wide variety of applied problems lead to equations of the form

$$
A x=y
$$

where $x$ is an unknown element of a certain infinite-dimensional space, $y$ is the data, supposed to be known up to a certain degree of noise and $A$ is a given operator used for modeling the system under consideration. Very often, $A^{\dagger}$, the Moore-Penrose generalized inverse of $A$, is unbounded and therefore the inverse problem (1) is ill-posed. It is well known that the best approximate solution, i.e. the least squares solution of minimum norm of (1) is $x^{\dagger}=A^{\dagger} y$ (see [2]). In the application of the least squares method to any concrete infinite-dimensional problem, discretization usually starts by choosing an increasing sequence of finite dimensional subspaces $X_{N}$ of $X$ whose union is dense in $X$, and then the sequence $\left\{x_{N}\right\}_{N=1}^{\infty}$, with $x_{N} \in X_{N}$ minimizing $\|A x-y\|^{2}$ over $X_{N}$, is constructed.

Luecke and Hickey [3] characterized the strong convergence to $x^{\dagger}$ of the sequence $\left\{x_{N}\right\}_{N=1}^{\infty}$ of least squares solutions of (1). They proved that if $A$ is a continuous linear operator and $y \in \mathcal{D}\left(A^{\dagger}\right)$, then $x_{N} \rightarrow x^{\dagger}$ if and only if $\limsup _{N \rightarrow \infty}\left\|x_{N}\right\| \leq\left\|x^{\dagger}\right\|$. They also found a sufficient condition for convergence which does not require of any a-priori knowledge about the norm of the true solution. More precisely, they showed that if $y \in \mathcal{D}\left(A^{\dagger}\right)$ then $x_{N} \rightarrow x^{\dagger}$ provided that $\sup _{N}\left\|\left(A_{N}^{\dagger}\right)^{*} x_{N}\right\|<\infty$ (or equivalently $\left.\sup \left\|\left(A_{N}^{*}\right)^{\dagger} x_{N}\right\|<\infty\right)$, where $A_{N} \doteq A P_{X_{N}}$ with $P_{X_{N}}$ being the orthogonal projection of $\stackrel{N}{X}$ onto $X_{N}$. Moreover they proved that if $A$ is compact and it has infinite rank, then this condition is not necessary for convergence. As a corollary they established that the uniform boundedness of the sequence $\left\{\left\|A_{N}^{\dagger}\right\|\right\}_{N=1}^{\infty}$ is also a sufficient condition for the strong convergence of $x_{N}$ to $x^{\dagger}$.

On the other hand, Luecke and Hickey [3] also showed that the boundedness of the sequence $\left\{\left\|x_{N}\right\|\right\}_{N=1}^{\infty}$ is not sufficient to guarantee strong convergence. However, Groetsch and Neubauer [4] proved that if $y \in \mathcal{D}\left(A^{\dagger}\right)$ then that condition is sufficient and, moreover necessary, for weak convergence. As a corollary of this result, they were also able to derive in a much more comprehensive manner the necessary and sufficient condition for strong convergence established earlier by Luecke and Hickey.

In order to guarantee convergence it is necessary that the subspaces $X_{N}$ be carefully chosen. For example, if $A$ is compact and the $X_{N}$ 's are the eigenspaces associated to the singular value decomposition of $A$, Luecke and Hickey [3] showed that one always has that $x_{N} \rightarrow x^{\dagger}$. They also proved that this convergence is guaranteed if $A$ is bounded and the subspaces $X_{N}$ are chosen so that they all reduce $A$ (in the sense that $A\left(X_{N}\right) \subset X_{N}$ and $\left.A\left(X_{N}^{\perp}\right) \subset X_{N}^{\perp}\right)$.

On the contrary, if the subspaces $X_{N}$ are carelessly chosen, e.g. when the $X_{N}$ are poorly or inadequately associated to the operator $A$, then almost anything can happen. For instance in [1] Seidman proved that if problem (1) is ill-posed, then without any 
additional assumptions on $x^{\dagger}$ it cannot be guaranteed that $x_{N} \rightarrow x^{\dagger}$ and that it is possible for $\left\|x_{N}-x^{\dagger}\right\| \rightarrow \infty$ to increase without bound. In this article we further show that if the subspaces $X_{N}$ are not carefully chosen, then not only things might go wrong with the application of the least squares method, but they can go arbitrarily bad. In fact, it will be shown that it is possible for $\left\|x_{N}-x^{\dagger}\right\|$ to increase at any arbitrary rate.

\section{Preliminaries}

The following result can be immediately derived from Example 3.1 in [1]:

Theorem 2.1. Let $X$ be an infinite-dimensional separable Hilbert space, $B \doteq\left\{e_{n}\right\}_{n=1}^{\infty}$ an orthonormal basis of $X$ and $X_{N} \doteq \operatorname{span}\left\{e_{1}, \ldots, e_{N}\right\}$. Then, there exist a linear, injective, compact, self-adjoint operator $A: X \rightarrow X$ whose range is dense in $X$ and $b \in \mathcal{R}(A)$ with $b=A x^{*}$ for some $x^{*} \in X$, such that if $x_{N}$ is the least squares solution of $A x=b$ in $X_{N}$, then $\left\|x_{N}-x^{*}\right\| \rightarrow \infty$.

Remark 2.2. The operator $A_{0}: X \rightarrow X$ of Example 3.1 in [1] is not necessarily selfadjoint. If $\left\{\left(\sigma_{n} ; v_{n}, u_{n}\right)\right\}$ denotes the singular system associated to the compact operator $A_{0}$ and $U: X \rightarrow X$ is the linear unitary operator defined by $U v_{n}=u_{n}$, then the linear operator $A \doteq U^{-1} A_{0}$, besides being compact, injective and having dense range, is self-adjoint and $\left\|A_{0} x-y\right\|=\left\|A x-U^{-1} y\right\|$ for every $y \in X$.

Corollary 2.3. Let $X$ and $Y$ be infinite-dimensional Hilbert spaces, $X$ separable, $B \doteq\left\{e_{n}\right\}_{n=1}^{\infty}$ an orthonormal basis of $X$ and $X_{N} \doteq \operatorname{span}\left\{e_{1}, \ldots, e_{N}\right\}$. Then, there exist a linear, injective, compact operator $A: X \rightarrow Y$ whose range is dense in $Y$ and an element $b \in \mathcal{R}(A), b=A x^{*}$ for some $x^{*} \in X$, such that if $x_{N}$ is the least squares solution of $A x=b$ in $X_{N}$, then $\left\|x_{N}-x^{*}\right\| \rightarrow \infty$.

Proof. From Theorem 2.1 there exist $A_{0}: X \rightarrow X$ and $b_{0} \in \mathcal{R}\left(A_{0}\right), b_{0}=A_{0} x^{*}$ for some $x^{*} \in X$, such that $\left\|x_{N}^{0}-x^{*}\right\| \rightarrow \infty$, where $x_{N}^{0}$ is the least squares solution of $A_{0} x=b_{0}$ in $X_{N}$. Let $V: X \rightarrow Y$ be an arbitrary unitary operator and let us define $A \doteq V A_{0}$ and $b \doteq V b_{0}$. Then it follows immediately that the operator $A: X \rightarrow Y$ is linear, compact, injective, with range dense in $Y$ and $b \in \mathcal{R}(A)$. Let $x_{N}$ be the least squares solution of $A x=b$ in $X_{N}$. Since $\|A x-b\|_{Y}=\left\|A_{0} x-b_{0}\right\|_{X}$, then it turns out that $x_{N}^{0}$ is also the least squares solution of $A x=b$ in $X_{N}$, that is, $x_{N}^{0}=x_{N}$. Therefore $\left\|x_{N}-x^{*}\right\| \rightarrow \infty$.

\section{Main Results}

In this section we will prove some results than are more general than those of Theorem 2.1 and Corollary 2.3. More precisely we will show that for any infinite-dimensional separable Hilbert space $X$, for any $b \in X, b \neq 0$, and for any arbitrarily given function $s: \mathbb{N} \rightarrow \mathbb{R}^{+}$it is possible to find an injective operator $A$ and a sequence of subspaces $X_{N} \subset X$ such that $\left\|x_{N}-A^{-1} b\right\| \geq s(N)$ for all $N \in \mathbb{N}$, where $x_{N}$ is the least squares solution of $A x=b$ in $X_{N}$. The following definitions will be needed. 
Definition 3.1. Let $X$ be an infinite-dimensional separable Hilbert space and $B \doteq$ $\left\{e_{n}\right\}_{n=1}^{\infty}$ an orthonormal basis of $X$. An element $x \in X$ is said to be "degenerate with respect to $B$ " if $x$ can be written as a finite linear combination of elements of $B$. Otherwise we say that $x$ is "non-degenerate with respect to $B$ ". In particular, if $\left\langle x, e_{n}\right\rangle \neq 0 \forall n \in \mathbb{N}$ then we say that $x$ is "strongly non-degenerate with respect to $B$ ". If $\left\langle x, e_{n}\right\rangle \neq 0$ for infinitely many $n$ 's, then we say that $x$ is "weakly non-degenerate with respect to $B "$. Clearly, if an element is strongly non-degenerate with respect to a basis, then it is also weakly non-degenerate with respect to the same basis.

The following results will also be needed later.

Lemma 3.2. Let $X$ be an infinite-dimensional separable Hilbert space and $b \in X, b \neq 0$. Then there exists an orthonormal basis $B$ of $X$ such that $b$ is strongly non-degenerate with respect to $B$.

Proof. Given $b \in X, b \neq 0$, let $\left\{g_{n}\right\}_{n=1}^{\infty}$ be an orthonormal basis of $[\operatorname{span}\{b\}]^{\perp}$ and define $f_{n} \doteq b+g_{n}, n \in \mathbb{N}$. Suppose that for a certain sequence of scalars $\alpha_{1}, \alpha_{2}, \ldots$, one has

$$
0=\sum_{n} \alpha_{n} f_{n}=\left(\sum_{n} \alpha_{n}\right) b+\sum_{n} \alpha_{n} g_{n} .
$$

Since $\{b\} \cup\left\{g_{n}\right\}_{n=1}^{\infty}$ is an orthogonal system, it is linearly independent and therefore, the equation above implies $\alpha_{n}=0$ for every $n \in \mathbb{N}$. Hence the $f_{n}$ 's are linearly independent.

We will now use the Gram-Schmidt process (see [5]) to construct an orthonormal system starting from the $f_{n}$ 's (note that $\left\langle f_{i}, f_{j}\right\rangle=\left\|b^{2}\right\|+\delta_{i, j}$ for $i, j \in \mathbb{N}$ ). For that, let us define

$$
e_{1} \doteq \frac{f_{1}}{\left\|f_{1}\right\|}
$$

and for $n \geq 2$, define $e_{n}$ recursively by

$$
e_{n} \doteq\left(f_{n}-\sum_{j=1}^{n-1}\left\langle f_{n}, e_{j}\right\rangle e_{j}\right)\left\|f_{n}-\sum_{j=1}^{n-1}\left\langle f_{n}, e_{j}\right\rangle e_{j}\right\|^{-1} .
$$

Then, it turns out that

$$
\operatorname{span}\left\{f_{n}\right\}_{n=1}^{\infty}=\operatorname{span}\left\{e_{n}\right\}_{n=1}^{\infty}
$$

and $\left\{e_{n}\right\}_{n=1}^{\infty}$ is an orthonormal system in $X$ (see [5]). Moreover, this system is complete in $X$. In fact, suppose there exists $y_{1} \in X$ such that $\left\langle y_{1}, e_{n}\right\rangle=0$ for every $n \in \mathbb{N}$. This implies that $\left\langle y_{1}, f_{n}\right\rangle=0$ for every $n \in \mathbb{N}$ by virtue of (2) and therefore

$$
\left\langle y_{1}, g_{n}\right\rangle=-\left\langle y_{1}, b\right\rangle \quad \text { for every } n \in \mathbb{N} \text {. }
$$

But, since $\left\{g_{n}\right\}_{n=1}^{\infty}$ is an orthonormal system in $X$, the sequence $\left\{\left\langle y_{1}, g_{n}\right\rangle\right\}_{n=1}^{\infty}$ must be in $\ell^{2}$. Thus, since by (3) that sequence is constant, we must have $\left\langle y_{1}, g_{n}\right\rangle=0$ for every $n \in \mathbb{N}$ which, again by virtue of (3), implies that also $\left\langle y_{1}, b\right\rangle=0$. Thus $y_{1}=0$ since $\left\{b, g_{1}, g_{2}, \ldots\right\}$ is complete in $X$. Hence $\left\{e_{n}\right\}_{n=1}^{\infty}$ is a complete orthonormal system in $X$. 
Next, we will show that $b$ is strongly non-degenerate with respect to the basis $B \doteq\left\{e_{n}\right\}_{n=1}^{\infty}$. On the contrary, suppose there exists $n^{*} \in \mathbb{N}$ such that $\left\langle e_{n^{*}}, b\right\rangle=0$ and $\left\langle e_{n}, b\right\rangle \neq 0$ for all $n<n^{*}$. Let $F_{n} \doteq \operatorname{span}\left\{f_{1}, \ldots, f_{n}\right\}$ and $E_{n} \doteq \operatorname{span}\left\{e_{1}, \ldots, e_{n}\right\}$. Since $\left\{f_{n}\right\}_{n=1}^{\infty}$ is a linearly independent set by the Gram-Schmidt procces, it turns out that $F_{n}=E_{n}$ for all $n \in \mathbb{N}$ (see [5]). Let now $P_{F_{n}}$ and $P_{E_{n}}$ denote the orthogonal projections of $X$ onto $F_{n}$ and $E_{n}$, respectively. Since $\left\langle e_{n^{*}}, b\right\rangle=0$, it follows that

$$
P_{F_{n^{*}}} b=P_{E_{n^{*}}} b=P_{E_{n^{*}-1}} b=P_{F_{n^{*}-1}} b,
$$

and therefore $\left\langle f_{n^{*}}, b\right\rangle=0$. But this contradicts the fact that $\left\langle f_{n^{*}}, b\right\rangle=\langle b, b\rangle+\left\langle g_{n^{*}}, b\right\rangle=$ $\|b\|^{2} \neq 0$. Thus, such an $n^{*}$ does not exist and $\left\langle e_{n}, b\right\rangle \neq 0$ for every $n \in \mathbb{N}$. We therefore conclude that $b$ is strongly non-degenerate with respect to the basis $B$ of $X$.

The following lemma is based upon the ideas presented in Example 3.1 in [1].

Lemma 3.3. Let $X$ be an infinite-dimensional separable Hilbert space, $B \doteq\left\{e_{n}\right\}_{n=1}^{\infty}$ an orthonormal basis of $X$ and let $\left\{\alpha_{n}\right\}_{n=1}^{\infty},\left\{\beta_{n}\right\}_{n=1}^{\infty}$ be two sequences in $\ell^{2}$ satisfying

$$
\alpha_{n} \neq 0 \forall n \in \mathbb{N}, \beta_{1}=0, \beta_{n} \neq 0 \forall n \geq 2 .
$$

Then, given $x=\sum_{n=1}^{\infty} \xi_{n} e_{n} \in X$, the linear operator $A: X \rightarrow X$ defined by

$$
A x \doteq \sum_{n=1}^{\infty}\left(\alpha_{n} \xi_{n}+\beta_{n} \xi_{1}\right) e_{n}
$$

is compact, injective and its range is dense in $X$.

Proof. We will first show that this operator is compact. For each $N \in \mathbb{N}$, let $\tilde{A}_{N}: X \rightarrow X_{N}$ be the operator defined by $\tilde{A}_{N} x \doteq \sum_{i=1}^{N}\left(\alpha_{i} \xi_{i}+\beta_{i} \xi_{1}\right) e_{i}$, where $x=\sum_{i=1}^{\infty} \xi_{i} e_{i} \in X$. For each $N \in \mathbb{N}, \tilde{A}_{N}$ is compact since it is clearly a finite rank operator. Moreover,

$$
\begin{aligned}
\left\|A x-\tilde{A}_{N} x\right\|^{2} & =\left\|\sum_{i=N+1}^{\infty}\left(\alpha_{i} \xi_{i}+\beta_{i} \xi_{1}\right) e_{i}\right\|^{2} \\
& =\sum_{i=N+1}^{\infty}\left(\alpha_{i} \xi_{i}+\beta_{i} \xi_{1}\right)^{2} \\
& \leq\|x\|^{2}\left(\sum_{i=N+1}^{\infty} \alpha_{i}^{2}+2 \sum_{i=N+1}^{\infty} \alpha_{i} \beta_{i}+\sum_{i=N+1}^{\infty} \beta_{i}^{2}\right)\left(\text { since } \xi_{i}^{2} \leq\|x\|^{2} \forall i \in \mathbb{N}\right) \\
& \leq\|x\|^{2}\left[\sum_{i=N+1}^{\infty} \alpha_{i}^{2}+2\left(\sum_{i=N+1}^{\infty} \alpha_{i}^{2}\right)^{\frac{1}{2}}\left(\sum_{i=N+1}^{\infty} \beta_{i}^{2}\right)^{\frac{1}{2}}+\sum_{i=N+1}^{\infty} \beta_{i}^{2}\right] \begin{array}{l}
\text { (by } \\
\text { Cauchy- } \\
\text { Schwartz }) .
\end{array}
\end{aligned}
$$

Since the sequences $\left\{\alpha_{n}\right\}_{n=1}^{\infty},\left\{\beta_{n}\right\}_{n=1}^{\infty}$ are both in $\ell^{2}$, it then follows that $\left\|A-\tilde{A}_{N}\right\| \rightarrow 0$ as $N \rightarrow \infty$. Hence, $A$, being uniform limit of compact operators, is itself compact.

We will now check that the operator $A$ is injective. Let $x=\sum_{i=1}^{\infty} \xi_{i} e_{i} \in X$ and suppose $A x=0$, then $\sum_{i=1}^{\infty} \alpha_{i} \xi_{i} e_{i}=-\sum_{i=1}^{\infty} \beta_{i} \xi_{1} e_{i}$. Equating coefficients of $e_{1}$ we have that $\alpha_{1} \xi_{1}=-\beta_{1} \xi_{1}$. Since $\beta_{1}=0$ and $\alpha_{1} \neq 0$ it follows that $\xi_{1}=0$. Similarly for all 
$i \geq 2, \alpha_{i} \xi_{i}=-\beta_{i} \xi_{1}=0$ which implies $\xi_{i}=0$ since $\xi_{1}=0$ and $\alpha_{i} \neq 0$. Hence $x=0$ and the operator $A$ is injective.

Next, we will show that $\mathcal{R}(A)$ is dense in $X$. Let $x=\sum_{i=1}^{\infty} \xi_{i} e_{i} \in X$. For each $N \in \mathbb{N}$, there are scalars $c_{1}, c_{2}, \ldots, c_{N}$ such that $z_{N} \doteq \sum_{i=1}^{N} c_{i} e_{i} \in X_{N}$ satisfies

$$
A z_{N}=\sum_{i=1}^{N}\left(\alpha_{i} c_{i}+\beta_{i} c_{1}\right) e_{i}=\sum_{i=1}^{N} \xi_{i} e_{i}
$$

In fact, equating the coefficients of $e_{i}, i=1, \ldots, N$, in this expression and recalling that $\beta_{1}=0$ and $\alpha_{i} \neq 0$ for all $i \in \mathbb{N}$, we immediately obtain $c_{1}=\frac{\xi_{1}}{\alpha_{1}}$ and $c_{i}=\alpha_{i}^{-1}\left(\xi_{i}-\frac{\beta_{i} \xi_{1}}{\alpha_{1}}\right)$ for $2 \leq i \leq N$. Moreover

$$
\left\|x-A z_{N}\right\|^{2}=\left\|\sum_{i=N+1}^{\infty} \xi_{i} e_{i}\right\|^{2}=\sum_{i=N+1}^{\infty} \xi_{i}^{2},
$$

and since $\left\{\xi_{i}\right\}_{i=1}^{\infty}$ is a sequence in $\ell^{2}$ we conclude that $\left\|x-A z_{N}\right\| \rightarrow 0$ as $N \rightarrow \infty$. Hence $\mathcal{R}(A)$ is dense in $X$. This finishes the proof of the lemma.

In the following result, we show that for any arbitrary infinite-dimensional separable Hilbert space $X$ and for any non zero element $b \in X$, there exists a linear operator $A$ such that the least squares solutions of the problem $A x=b$ in $X_{N}$ diverge from the exact solution with arbitrarily large speed. More precisely we have the following Theorem.

Theorem 3.4. Let $X$ be an infinite-dimensional separable Hilbert space and $s: \mathbb{N} \rightarrow$ $\mathbb{R}^{+}$an arbitrary nonnegative increasing function. Then for each $b \in X, b \neq 0$ there exist an increasing sequence of subspaces $X_{N}$ whose union is dense in $X$ and a linear, compact, injective operator $A=A(b, s): X \rightarrow X$, whose range is dense in $X$, such that $b=A x^{*}$ for some $x^{*} \in X$ and, if $x_{N}$ denotes the least squares solution of $A x=b$ in $X_{N}$, then $\left\|x_{N}-x^{*}\right\| \geq s(N)$ for every $N \in \mathbb{N}$.

Proof. Let $b \in X, b \neq 0$. From Lemma 3.2 there exists an orthonormal basis $B \doteq\left\{e_{n}\right\}_{n=1}^{\infty}$ of $X$ such that $b$ is strongly non-degenerate with respect to $B$. Define $X_{N} \doteq \operatorname{span}\left\{e_{1}, \ldots, e_{N}\right\}$, let $\left\{\alpha_{n}\right\}_{n=1}^{\infty},\left\{\beta_{n}\right\}_{n=1}^{\infty}$ be any two sequences in $\ell^{2}$ satisfying (4) and let $A$ be the linear operator defined in (5). By virtue of Lemma $3.3 A$ is compact, injective and its range is dense in $X$.

Next we will show that given any element $b \in X$, strongly non-degenerate with respect to $B$, i.e. such that $b_{n} \doteq\left\langle b, e_{n}\right\rangle \neq 0$ for every $n \in \mathbb{N}$, it is possible to choose the sequences $\left\{\alpha_{n}\right\}_{n=1}^{\infty},\left\{\beta_{n}\right\}_{n=1}^{\infty} \in \ell^{2}$ in such a way that, besides satisfying (4), one has $b \in \mathcal{R}(A)$ and, if $x_{N}$ denotes the least squares solution of $A x=b$ in $X_{N}$, then $\left\|x_{N}-A^{-1} b\right\| \geq s(N)$ for every $N \in \mathbb{N}$.

The sequences $\left\{\alpha_{n}\right\}_{n=1}^{\infty}$ and $\left\{\beta_{n}\right\}_{n=1}^{\infty}$ shall be constructed recursively, as follows:

Step 1: Choose $\alpha_{1}$ arbitrary, $\alpha_{1} \neq 0$ and $\beta_{1} \doteq 0$. 
Step 2: $\alpha_{2} \doteq \frac{1}{2}$,

$$
\beta_{2} \doteq \begin{cases}\frac{\alpha_{1} b_{2}}{2 b_{1}}, & \text { if } b_{2}=\frac{\alpha_{2}}{2^{\frac{2}{2}+1}}=\frac{1}{8} \\ \frac{\alpha_{1}}{b_{1}}\left(b_{2}-\frac{\alpha_{2}}{2^{\frac{2}{2}+1}}\right)=\frac{\alpha_{1}}{b_{1}}\left(b_{2}-\frac{1}{8}\right), & \text { in other case }\end{cases}
$$

It suffices to choose $\beta_{2} \neq 0$ such that $\frac{b_{1} \beta_{2}}{\alpha_{1}} \in\left(b_{2}-\frac{\alpha_{2}}{2^{\frac{2}{2}}}, b_{2}\right)$.

Step 3: $\alpha_{4} \doteq \frac{1}{4}$,

$$
\beta_{4} \doteq \begin{cases}\frac{\alpha_{1} b_{4}}{2 b_{1}}, & \text { if } b_{4}=\frac{\alpha_{4}}{2^{\frac{4}{2}+1}}=\frac{1}{32} \\ \frac{\alpha_{1}}{b_{1}}\left(b_{4}-\frac{\alpha_{4}}{2^{\frac{4}{2}+1}}\right)=\frac{\alpha_{1}}{b_{1}}\left(b_{4}-\frac{1}{32}\right), & \text { in other case }\end{cases}
$$

Also here, it suffices to choose $\beta_{4} \neq 0$ such that $\frac{b_{1} \beta_{4}}{\alpha_{1}} \in\left(b_{4}-\frac{\alpha_{4}}{2^{\frac{4}{2}}}, b_{4}\right)$.

Step 4: $\alpha_{6} \doteq \frac{1}{6}$,

$$
\beta_{6} \doteq \begin{cases}\frac{\alpha_{1} b_{6}}{2 b_{1}}, & \text { if } b_{6}=\frac{\alpha_{6}}{2^{\frac{6}{2}+1}}=\frac{1}{96} \\ \frac{\alpha_{1}}{b_{1}}\left(b_{6}-\frac{\alpha_{6}}{2^{\frac{6}{2}+1}}\right)=\frac{\alpha_{1}}{b_{1}}\left(b_{6}-\frac{1}{96}\right), & \text { in other case }\end{cases}
$$

As before, it suffices to choose $\beta_{6} \neq 0$ such that $\frac{b_{1} \beta_{6}}{\alpha_{1}} \in\left(b_{6}-\frac{\alpha_{6}}{2^{\frac{6}{2}}}, b_{6}\right)$. For simplicity let us denote $K \doteq \alpha_{1}^{2}\left[1+b_{1}^{-2}\left(\frac{5}{4}\|b\|^{2}+\sqrt{2}\|b\|+2\right)\right]$.

Step 5: $\alpha_{3}$ is chosen according to the sign of $b_{3}$ as follows:

- If $b_{3}<0$, then

$$
\alpha_{3} \doteq \min \left\{\frac{1}{3} ;\left|\frac{\alpha_{1} b_{3} \beta_{6}}{b_{1}}\right|\left(b_{6}-\frac{\beta_{6} b_{1}}{\alpha_{1}}\right)[K s(4)]^{-1}\right\}>0 .
$$

Note that $\alpha_{3}>0$ since $K>0$ and by the election of $\beta_{6}$ made in step 4 , we have that $b_{6}-\frac{\beta_{6} b_{1}}{\alpha_{1}}>0$.

- If $b_{3}>0$, then we define

$$
\alpha_{3} \doteq \min \left\{\frac{1}{3} ; 2^{\frac{3}{2}} b_{3} ; b_{3}\left[\frac{K s(4)}{\left|\frac{\alpha_{1} \beta_{6}}{b_{1}}\right|\left(b_{6}-\frac{\beta_{6} b_{1}}{\alpha_{1}}\right)}+2^{-\frac{3}{2}}\right]^{-1}\right\}>0 .
$$

In (6) and (7), it actually suffices to pick $\alpha_{3}$ as any positive number less or equal than the respective minimum.

\section{Step 6:}

$$
\beta_{3} \doteq \begin{cases}\frac{\alpha_{1} b_{3}}{2 b_{1}}, & \text { if } b_{3}=\frac{\alpha_{3}}{2^{\frac{3}{2}+1}} \\ \frac{\alpha_{1}}{b_{1}}\left(b_{3}-\frac{\alpha_{3}}{2^{\frac{3}{2}+1}}\right), & \text { in other case }\end{cases}
$$

Here again it suffices to choose $\beta_{3} \neq 0$ such that $\frac{b_{1} \beta_{3}}{\alpha_{1}} \in\left(b_{3}-\frac{\alpha_{3}}{2^{\frac{3}{2}}}, b_{3}\right)$.

Steps similar to 4,5 and 6 are followed to construct $\alpha_{2 j}, \beta_{2 j}$ and $\alpha_{2 j-3}, \beta_{2 j-3}$ for $j \geq 4$. More precisely: 
Step 7: for all $j \geq 4$ define

$$
\begin{aligned}
& \alpha_{2 j} \doteq \frac{1}{2 j} \text {, } \\
& \beta_{2 j} \doteq \begin{cases}\frac{\alpha_{1} b_{2 j}}{2 b_{1}}, & \text { if } b_{2 j}=\frac{\alpha_{2 j}}{2^{j+1}}, \\
\frac{\alpha_{1}}{b_{1}}\left(b_{2 j}-\frac{\alpha_{2 j}}{2^{j+1}}\right), & \text { in other case. }\end{cases}
\end{aligned}
$$

Here, it suffices to choose $\beta_{2 j} \neq 0$ such that $\frac{b_{1} \beta_{2 j}}{\alpha_{1}} \in\left(b_{2 j}-\frac{\alpha_{2 j}}{2^{j}}, b_{2 j}\right)$.

Step 8: For all $j \geq 4, \alpha_{2 j-3}$ is chosen according to the sign of $b_{2 j-3}$ as follows:

- If $b_{2 j-3}<0$, define

$$
\alpha_{2 j-3} \doteq \min \left\{\frac{1}{2 j-3} ;\left|\frac{\alpha_{1} b_{2 j-3} \beta_{2 j}}{b_{1}}\right|\left(b_{2 j}-\frac{\beta_{2 j} b_{1}}{\alpha_{1}}\right)[K s(2 j-2)]^{-1}\right\}>0 \text {. }
$$

Note that $\alpha_{2 j-3}>0$ since, by the choice of $\beta_{2 j}$ in step 7, we have that $b_{2 j}-\frac{\beta_{2 j} b_{1}}{\alpha_{1}}>0$.

- If $b_{2 j-3}>0$, define

$$
\alpha_{2 j-3} \doteq \min \left\{\frac{1}{2 j-3} ; 2^{\frac{2 j-3}{2}} b_{2 j-3} ; b_{2 j-3}\left[\frac{K s(2 j-2)}{\left|\frac{\alpha_{1} \beta_{2 j}}{b_{1}}\right|\left(b_{2 j}-\frac{\beta_{2 j} b_{1}}{\alpha_{1}}\right)}+2^{-\frac{2 j-3}{2}}\right]^{-1}\right\}>0 \text {. }
$$

In (8) and (9) it suffices to choose $\alpha_{2 j-3}$ to be any positive number less or equal than the respective minimum.

Finally, define

$$
\beta_{2 j-3} \doteq \begin{cases}\frac{\alpha_{1} b_{2 j-3}}{2 b_{1}}, & \text { if } b_{2 j-3}=\frac{\alpha_{2 j-3}}{2^{2 j-3}+1} \\ \frac{\alpha_{1}}{b_{1}}\left(b_{2 j-3}-\frac{\alpha_{2 j-3}}{2^{\frac{2 j-3}{2}+1}}\right), & \text { in other case. }\end{cases}
$$

It suffices to choose $\beta_{2 j-3} \neq 0$ such that $\frac{b_{1} \beta_{2 j-3}}{\alpha_{1}} \in\left(b_{2 j-3}-\frac{\alpha_{2 j-3}}{2^{\frac{2 j-3}{2}}}, b_{2 j-3}\right)$.

Figure 1 depicts the recursion order used for the construction of the sequences $\left\{\alpha_{n}\right\}_{n=1}^{\infty}$ and $\left\{\beta_{n}\right\}_{n=1}^{\infty}$. It is easy to check that these sequences satisfy the conditions (4) and are both in $\ell^{2}$. In fact, $\sum_{n=1}^{\infty} \alpha_{n}^{2} \leq \sum_{n=1}^{\infty} \frac{1}{n^{2}}<\infty$, since $0<\alpha_{n} \leq \frac{1}{n}$ for every $n \in \mathbb{N}$ due to the choice of the $\alpha_{n}$ 's. On the other hand, by our choice of the sequence $\left\{\beta_{n}\right\}_{n=1}^{\infty}$ it follows that

$$
\begin{aligned}
\sum_{n=1}^{\infty} \beta_{n}^{2} & \leq \sum_{n=1}^{\infty}\left(\frac{\alpha_{1} b_{n}}{2 b_{1}}\right)^{2}+\sum_{n=1}^{\infty}\left(\frac{\alpha_{1}}{b_{1}}\right)^{2}\left(b_{n}-\frac{\alpha_{n}}{2^{\frac{n}{2}+1}}\right)^{2} \\
& =\frac{\alpha_{1}^{2}}{b_{1}^{2}}\left[\frac{1}{4} \sum_{n=1}^{\infty} b_{n}^{2}+\sum_{n=1}^{\infty}\left(b_{n}^{2}-\frac{b_{n} \alpha_{n}}{2^{\frac{n}{2}}}+\frac{\alpha_{n}^{2}}{2^{n+2}}\right)\right] \\
& \leq \frac{\alpha_{1}^{2}}{b_{1}^{2}}\left[\frac{5}{4}\|b\|^{2}+\left(\sum_{n=1}^{\infty} b_{n}^{2}\right)^{\frac{1}{2}}\left(\sum_{n=1}^{\infty} \frac{\alpha_{n}^{2}}{2^{n}}\right)^{\frac{1}{2}}+\sum_{n=1}^{\infty} \alpha_{n}^{2}\right] \quad \text { (by Cauchy-Schwartz and } \\
& \leq \frac{\alpha_{1}^{2}}{b_{1}^{2}}\left[\frac{5}{4}\|b\|^{2}+\|b\|\left(\sum_{n=1}^{\infty} \frac{1}{n^{2}}\right)^{\frac{1}{2}}+\sum_{n=1}^{\infty} \frac{1}{n^{2}}\right] \quad\left(\text { since } \alpha_{n} \leq \frac{1}{n} \text { and } 2^{n}>1 \forall n \in \mathbb{N}\right)
\end{aligned}
$$




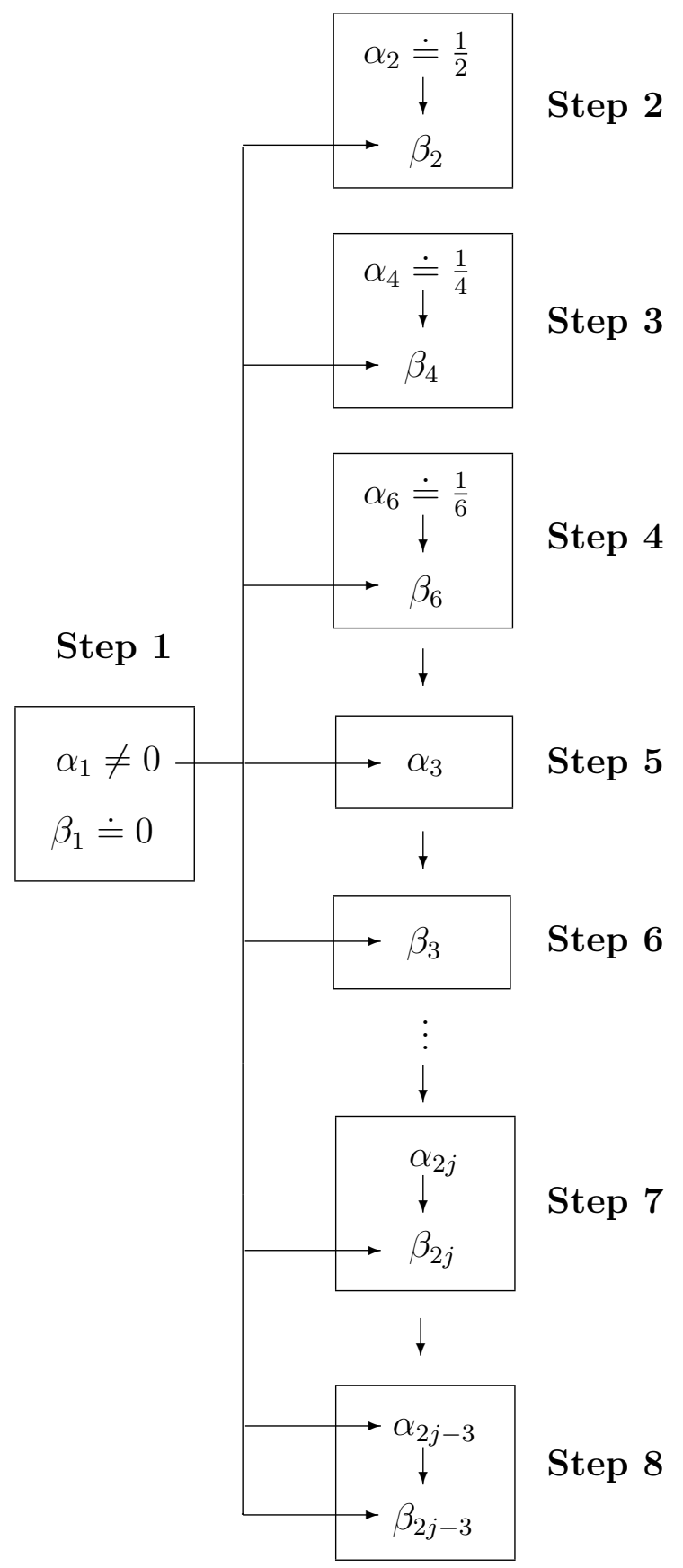

Figure 1. Recursion order used for the construction of the sequences $\left\{\alpha_{n}\right\}$ and $\left\{\beta_{n}\right\}$.

$$
\begin{aligned}
& \leq \frac{\alpha_{1}^{2}}{b_{1}^{2}}\left(\frac{5}{4}\|b\|^{2}+\sqrt{2}\|b\|+2\right) \quad\left(\text { since } \sum_{n=1}^{\infty} \frac{1}{n^{2}}=\frac{\pi^{2}}{6}<2\right) \\
& =K-\alpha_{1}^{2}<\infty .
\end{aligned}
$$

We will check now that $b \in \mathcal{R}(A)$, i.e., that there exists $x^{*}=\sum_{n=1}^{\infty} \xi_{n}^{*} e_{n} \in X$ such that $A x^{*}=b$. In order to do that, we will show that the system of equations derived from the equality $\sum_{n=1}^{\infty}\left(\alpha_{n} \xi_{n}^{*}+\beta_{n} \xi_{1}^{*}\right) e_{n}=\sum_{n=1}^{\infty} b_{n} e_{n}$, has a unique solution 
$\left\{\xi_{n}^{*}\right\} \in \ell^{2}$. Equating first the coefficients of $e_{1}$ we have $\alpha_{1} \xi_{1}^{*}+\beta_{1} \xi_{1}^{*}=b_{1}$. Since $\beta_{1}=0$ it follows that $\xi_{1}^{*}=\frac{b_{1}}{\alpha_{1}}$. Similarly, equating the coefficients of $e_{n}$ for $n \geq 2$ it follows that $\alpha_{n} \xi_{n}^{*}+\beta_{n} \xi_{1}^{*}=b_{n}$, which implies

$$
\xi_{n}^{*}=\left(b_{n}-\frac{b_{1} \beta_{n}}{\alpha_{1}}\right) \alpha_{n}^{-1} .
$$

Hence,

$$
\begin{aligned}
\sum_{n=1}^{\infty} \xi_{n}^{* 2} & =\left(\frac{b_{1}}{\alpha_{1}}\right)^{2}+\sum_{n=2}^{\infty}\left(b_{n}-\frac{b_{1} \beta_{n}}{\alpha_{1}}\right)^{2} \alpha_{n}^{-2} \\
& =\left(\frac{b_{1}}{\alpha_{1}}\right)^{2}+\sum_{\substack{n=2 \\
b_{n}=\frac{\alpha_{n}}{2^{\frac{n}{2}+1}}}}^{\infty}\left(b_{n}-\frac{b_{1} \beta_{n}}{\alpha_{1}}\right)^{2} \alpha_{n}^{-2}+\sum_{\substack{n=2 \\
b_{n} \neq \frac{\alpha_{n}}{2^{\frac{n}{2}+1}}}}^{\infty}\left(b_{n}-\frac{b_{1} \beta_{n}}{\alpha_{1}}\right)^{2} \alpha_{n}^{-2} \\
& =\left(\frac{b_{1}}{\alpha_{1}}\right)^{2}+\sum_{\substack{n=2 \\
b_{n}=\frac{\alpha_{n}}{2^{\frac{n}{2}+1}}}}^{\infty}\left(b_{n}-\frac{b_{n}}{2}\right)^{2} \alpha_{n}^{-2}+\sum_{\substack{n=2 \\
b_{n} \neq \frac{\alpha_{n}}{2^{\frac{n}{2}+1}}}}^{\infty}\left(\frac{\alpha_{n}}{2^{\frac{n}{2}+1}}\right)^{2} \alpha_{n}^{-2} \quad \text { (by our choice of } \\
& \leq\left(\frac{b_{1}}{\alpha_{1}}\right)^{2}+\sum_{n=2}^{\infty} \frac{1}{2^{n+4}}+\sum_{n=2}^{\infty} \frac{1}{2^{n+2}}<\infty .
\end{aligned}
$$

Thus $\left\{\xi_{n}^{*}\right\}_{n=1}^{\infty} \in \ell^{2}, x^{*}=\sum_{n=1}^{\infty} \xi_{n}^{*} e_{n} \in X$ and $b=A x^{*} \in \mathcal{R}(A)$.

Let now $x_{N}$ be the least squares solution of $A x=b$ in $X_{N}$, and write $x_{N}=$ $\sum_{n=1}^{N} \xi_{n} e_{n}$. We will show that $\left\|x_{N}-x^{*}\right\| \geq s(N)$ for every $N \in \mathbb{N}$. Defining the functional $\mathcal{J}\left(x_{N}\right) \doteq \frac{1}{2}\left\|A x_{N}-A x^{*}\right\|^{2}$, and equating to zero each one of its partial derivatives with respect to $\xi_{i}, i=1, \ldots, N$ (for the sake of brevity we skip the details here) it follows immediately that

$$
\begin{aligned}
& \xi_{1}=\xi_{1}^{*}+\frac{\sum_{n=N+1}^{\infty} \alpha_{n} \beta_{n} \xi_{n}^{*}}{\alpha_{1}^{2}+\sum_{n=N+1}^{\infty} \beta_{n}^{2}}, \\
& \xi_{n}=\xi_{n}^{*}-\frac{\beta_{n}}{\alpha_{n}}\left(\xi_{1}-\xi_{1}^{*}\right), \text { for } 2 \leq n \leq N .
\end{aligned}
$$

Therefore,

$$
\begin{aligned}
\left\|x_{N}-x^{*}\right\|^{2} & =\frac{\left(\sum_{n=N+1}^{\infty} \alpha_{n} \beta_{n} \xi_{n}^{*}\right)^{2}}{\left(\alpha_{1}^{2}+\sum_{n=N+1}^{\infty} \beta_{n}^{2}\right)^{2}}\left(1+\sum_{n=2}^{N} \frac{\beta_{n}^{2}}{\alpha_{n}^{2}}\right)+\sum_{n=N+1}^{\infty} \xi_{n}^{* 2} \\
& \geq \frac{\left(\sum_{n=N+1}^{\infty} \alpha_{n} \beta_{n} \xi_{n}^{*}\right)^{2}}{\left(\alpha_{1}^{2}+\sum_{n=N+1}^{\infty} \beta_{n}^{2}\right)^{2}} \sum_{n=2}^{N} \frac{\beta_{n}^{2}}{\alpha_{n}^{2}} .
\end{aligned}
$$

Hence,

(a) For $N$ even,

$$
\left\|x_{N}-x^{*}\right\|^{2} \geq \frac{\left(\alpha_{N+2} \beta_{N+2} \xi_{N+2}^{*}\right)^{2}}{\left(\alpha_{1}^{2}+\sum_{n=N+1}^{\infty} \beta_{n}^{2}\right)^{2}} \frac{\beta_{N-1}^{2}}{\alpha_{N-1}^{2}}
$$




$$
=\frac{\beta_{N+2}^{2}\left(b_{N+2}-\frac{b_{1} \beta_{N+2}}{\alpha_{1}}\right)^{2}}{\left(\alpha_{1}^{2}+\sum_{n=N+1}^{\infty} \beta_{n}^{2}\right)^{2}} \frac{\beta_{N-1}^{2}}{\alpha_{N-1}^{2}} \quad \begin{array}{ll}
\xi_{N+2}^{*} & \text { by }(12)) .
\end{array}
$$

(a.1) If $b_{N-1}<0$, then by the election of the $\beta_{n}$ 's we have $\frac{b_{1} \beta_{N-1}}{\alpha_{1}}<b_{N-1}<0$ and therefore $\beta_{N-1}^{2}>\left(\frac{\alpha_{1} b_{N-1}}{b_{1}}\right)^{2}$, which, together with our choice of $\alpha_{N-1}$ (see (8) in step 8) implies that

$$
\begin{aligned}
\left\|x_{N}-x^{*}\right\|^{2} & \geq \frac{[s(N)]^{2} K^{2}}{\left(\alpha_{1}^{2}+\sum_{n=N+1}^{\infty} \beta_{n}^{2}\right)^{2}} \\
& \geq \frac{[s(N)]^{2}\left(\alpha_{1}^{2}+\sum_{n=1}^{\infty} \beta_{n}^{2}\right)^{2}}{\left(\alpha_{1}^{2}+\sum_{n=N+1}^{\infty} \beta_{n}^{2}\right)^{2}} \quad(\text { from }(11)) \\
& \geq[s(N)]^{2} .
\end{aligned}
$$

(a.2) If $b_{N-1}>0$, since $\alpha_{N-1}$ was chosen so that $\alpha_{N-1} \leq 2^{\frac{N-1}{2}} b_{N-1}$ (see (9) in step 8) and recalling our choice of $\beta_{N-1}$ (see (10)), it follows that $0 \leq b_{N-1}-\frac{\alpha_{N-1}}{2^{\frac{N-1}{2}}}<\frac{b_{1} \beta_{N-1}}{\alpha_{1}}$. Then, $\beta_{N-1}^{2}>\frac{\alpha_{1}^{2}}{b_{1}^{2}}\left(b_{N-1}-\frac{\alpha_{N-1}}{2^{\frac{N-1}{2}}}\right)^{2}$. Substituting with this into (14) we finally obtain that

$$
\begin{aligned}
\left\|x_{N}-x^{*}\right\|^{2} & \geq \frac{\left[\beta_{N+2}\left(b_{N+2}-\frac{b_{1} \beta_{N+2}}{\alpha_{1}}\right)\right]^{2}}{\left(\alpha_{1}^{2}+\sum_{n=N+1}^{\infty} \beta_{n}^{2}\right)^{2}} \frac{\alpha_{1}^{2}}{b_{1}^{2}}\left(b_{N-1}-\frac{\alpha_{N-1}}{2^{\frac{N-1}{2}}}\right)^{2} \frac{1}{\alpha_{N-1}^{2}} \\
& =\frac{\left[\beta_{N+2}\left(b_{N+2}-\frac{b_{1} \beta_{N+2}}{\alpha_{1}}\right)\right]^{2}}{\left(\alpha_{1}^{2}+\sum_{n=N+1}^{\infty} \beta_{n}^{2}\right)^{2}} \frac{\alpha_{1}^{2}}{b_{1}^{2}}\left(\frac{b_{N-1}}{\alpha_{N-1}}-\frac{1}{2^{\frac{N-1}{2}}}\right)^{2} \\
& \geq \frac{[s(N)]^{2} K^{2}}{\left(\alpha_{1}^{2}+\sum_{n=N+1}^{\infty} \beta_{n}^{2}\right)^{2}}(\text { from }(9)) \\
& \geq \frac{[s(N)]^{2}}{\left(\alpha_{1}^{2}+\sum_{n=N+1}^{\infty} \beta_{n}^{2}\right)^{2}}\left(\alpha_{1}^{2}+\sum_{n=1}^{\infty} \beta_{n}^{2}\right)^{2} \quad(\text { from }(11)) \\
& \geq[s(N)]^{2} .
\end{aligned}
$$

(b) For $N$ odd, it follows from (13) that

$$
\left\|x_{N}-x^{*}\right\|^{2} \geq \frac{\left(\alpha_{N+3} \beta_{N+3} \xi_{N+3}^{*}\right)^{2}}{\left(\alpha_{1}^{2}+\sum_{n=N+1}^{\infty} \beta_{n}^{2}\right)^{2}} \frac{\beta_{N}^{2}}{\alpha_{N}^{2}} .
$$

Following similar steps as in the previous case ( $N$ even) it follows easily that now $\left\|x_{N}-x^{*}\right\|^{2} \geq[s(N+1)]^{2} \geq[s(N)]^{2}$, where the last inequality follows from the fact that $s$ is an increasing function. This completes the proof of the Theorem.

Later on, in Corollary 3.7, we will see that under certain general additional assumptions on $b$, the operator $A$ in the previous Theorem can be constructed to further be self-adjoint.

For our next Corollary, the following Lemma will be needed. 
Lemma 3.5. Let $X$ be a Hilbert space, $H$ a closed subspace of $X, A: X \rightarrow X$ a bounded linear operator, $M$ a closed subspace of $X$ such that $M$ and $M^{\perp}$ are invariant under $A$ and $b \in M$. If $x^{*}$ is the least squares solution of $A x=b$ in $H$, then the least squares solution of $A x=b$ in $H \cap M$ is $P_{M} x^{*}$, where $P_{M}$ is the orthogonal projection of $X$ onto $M$.

Proof. Let us suppose that $P_{M} x^{*}$ is not the least squares solution of $A x=b$ in $H \cap M$. Then, there exists $z \in H \cap M, z \neq P_{M} x^{*}$, such that

$$
\|A z-b\|<\left\|A P_{M} x^{*}-b\right\| \text {. }
$$

Let $\widetilde{x^{*}} \doteq z+P_{M \perp} x^{*} \in H$. Since $z \neq P_{M} x^{*}$, it turns out that $\widetilde{x^{*}} \neq x^{*}$. Then, since $b \in M, z \in M$ and $M, M^{\perp}$ are both invariant under $A$, we have that

$$
\begin{aligned}
\left\|A \widetilde{x^{*}}-b\right\|^{2} & =\left\|A z+A P_{M^{\perp}} x^{*}-b\right\|^{2} \\
& =\|A z-b\|^{2}+\left\|A P_{M^{\perp}} x^{*}\right\|^{2} \\
& <\left\|A P_{M} x^{*}-b\right\|^{2}+\left\|A P_{M^{\perp}} x^{*}\right\|^{2} \quad(\text { from (15)) } \\
& =\left\|A x^{*}-b\right\|^{2},
\end{aligned}
$$

which is a contradiction since $\widetilde{x^{*}} \in H$ and $x^{*}$ is the least squares solution of $A x=b$ in $H$. Hence, $P_{M} x^{*}$ is the least squares solution of $A x=b$ in $H \cap M$.

In actual applications of the least squares method, the basis $B=\left\{e_{n}\right\}_{n=1}^{\infty}$ of $X$ is usually given and $X_{N}$ is chosen as $\operatorname{span}\left\{e_{1}, \ldots, e_{N}\right\}$. In the next Corollary, we shown that in this case, for any $b \in X$ weakly non-degenerate with respect to that basis and for any function $s: \mathbb{N} \rightarrow \mathbb{R}^{+}$as before, it is also possible to construct an operator $A=A(b, s)$ such that the least squares solutions in $X_{N}$ of $A x=b$ diverge from the exact solution with speed greater or equal than $s$.

Corollary 3.6. Let $X$ be an infinite-dimensional separable Hilbert space, $s: \mathbb{N} \rightarrow \mathbb{R}^{+}$ an arbitrary nonnegative increasing function, $B \doteq\left\{e_{n}\right\}_{n=1}^{\infty}$ an orthonormal basis of $X$, $b \in X$ weakly non-degenerate with respect to $B$ and $X_{N} \doteq \operatorname{span}\left\{e_{1}, \ldots, e_{N}\right\}$. Then there exists a linear, compact, injective operator $A=A(b, s): X \rightarrow X$, whose range is dense in $X$, such that $b=A \hat{x}$ for some $\hat{x} \in X$ and, if $x_{N}$ is the least squares solution of $A x=b$ in $X_{N}$, then $\left\|x_{N}-\hat{x}\right\| \geq s(N)$ for every $N \in \mathbb{N}$.

Proof. Let us define

$$
\begin{aligned}
& \Delta \doteq\left\{n \in \mathbb{N}:\left\langle b, e_{n}\right\rangle \neq 0\right\}, \quad \Gamma \doteq \mathbb{N} \backslash \Delta, \\
& B^{\Delta} \doteq\left\{e_{n_{j}}: n_{j} \in \Delta\right\}, \quad B^{\Gamma} \doteq\left\{e_{m_{j}}: m_{j} \in \Gamma\right\},
\end{aligned}
$$

and let $X_{b} \doteq \overline{\operatorname{span} B^{\Delta}}$. Clearly,

$$
\overline{\operatorname{span} B^{\Gamma}}=X_{b}^{\perp}
$$

and $b$ is strongly non-degenerate with respect to the basis $B^{\Delta}$ of $X_{b}$. Since $X_{b}$ is an infinite-dimensional separable Hilbert space, Theorem 3.4 implies that there exists a 
compact, injective, linear operator $A^{\Delta}: X_{b} \rightarrow X_{b}$ whose range is dense in $X_{b}$ such that $b=A^{\Delta} \hat{x}$ for some $\hat{x} \in X_{b}$ and if $x_{M}^{\Delta}$ is the least squares solution of $A^{\Delta} x=b$ in

$$
X_{M}^{\Delta} \doteq \operatorname{span}\left\{e_{n_{j}}\right\}_{j=1}^{M} \subset X_{b}
$$

then

$$
\left\|x_{M}^{\Delta}-\hat{x}\right\| \geq \tilde{s}(M) \forall M \in \mathbb{N}
$$

where $\tilde{s}: \mathbb{N} \rightarrow \mathbb{R}^{+}$is defined by $\tilde{s}(j) \doteq s\left(n_{j+1}\right)$.

Next, the operator $A$ will be constructed by appropriately extending $A^{\Delta}$ to the whole space $X$. The way in which this extension is made depends upon the cardinality of the set $\Gamma$ defined in (16).

Case I: The set $\Gamma$ is not finite. In this case $X_{b}^{\perp}$ is an infinite-dimensional separable Hilbert space. Let $\left\{\gamma_{n}\right\}_{n=1}^{\infty}$ be any sequence in $\ell^{2}$ such that $\gamma_{n} \neq 0 \forall n \in \mathbb{N}$ and define $\tilde{b} \doteq \sum_{n \in \Gamma} \gamma_{n} e_{n}$. Clearly $\tilde{b}$ is strongly non-degenerate with respect to the basis $B^{\Gamma}$ of $X_{b}^{\perp}$. By virtue of Theorem 3.4, there exists a compact, injective linear operator $A^{\Gamma}: X_{b}^{\perp} \rightarrow X_{b}^{\perp}$ whose range is dense in $X_{b}^{\perp}$ such that $\tilde{b}=A^{\Gamma} \tilde{x}$ for some $\tilde{x} \in X_{b}^{\perp}$. We then define $A$ as follows:

$$
A x \doteq A^{\Delta} x_{1}+A^{\Gamma} x_{2},
$$

where $x=x_{1}+x_{2}$ with $x_{1} \in X_{b}$ and $x_{2} \in X_{b}^{\perp}$.

Case II: The set $\Gamma$ is finite. In this case we simply define $A$ as:

$$
A x \doteq A^{\Delta} x_{1}+x_{2},
$$

where as before $x=x_{1}+x_{2}$, with $x_{1} \in X_{b}$ and $x_{2} \in X_{b}^{\perp}$.

It is clear that in both cases the operator $A$ so defined is linear. Let us prove now that this operator is also injective, compact and that its range is dense in $X$.

Let us first consider case I (see (20)). If $A x=0$, then $A^{\Delta} x_{1}=-A^{\Gamma} x_{2}$. Since $A^{\Delta} x_{1} \in X_{b}$ and $-A^{\Gamma} x_{2} \in X_{b}^{\perp}$ we have that $A^{\Delta} x_{1}=-A^{\Gamma} x_{2}=0$ and therefore $x_{1}=x_{2}=0$ since $A^{\Delta}$ and $A^{\Gamma}$ are both injective operators. Thus, $x=x_{1}+x_{2}=0$ and therefore $A$ is injective.

Let us show now that $\mathcal{R}(A)$ is dense in $X$. By definition of $A$ and due to the fact that $\mathcal{R}\left(A^{\Delta}\right) \subset X_{b}$ and $\mathcal{R}\left(A^{\Gamma}\right) \subset X_{b}^{\perp}$, it follows that $\mathcal{R}\left(A^{\Delta}\right)$ and $\mathcal{R}\left(A^{\Gamma}\right)$ are subspaces of $X$ which are orthogonal to each other. Therefore $\mathcal{R}(A)=\mathcal{R}\left(A^{\Delta}\right) \oplus \mathcal{R}\left(A^{\Gamma}\right)$. Then,

$$
\begin{aligned}
\overline{\mathcal{R}(A)} & =\overline{\mathcal{R}\left(A^{\Delta}\right)} \oplus \overline{\mathcal{R}\left(A^{\Gamma}\right)} \\
& =\left(X_{b} \cap \overline{\mathcal{R}\left(A^{\Delta}\right)}\right) \oplus\left(X_{b}^{\perp} \cap \overline{\mathcal{R}\left(A^{\Gamma}\right)}\right) \quad\left(\text { since } \mathcal{R}\left(A^{\Delta}\right) \subset X_{b} \text { and } \mathcal{R}\left(A^{\Gamma}\right) \subset X_{b}^{\perp}\right) \\
& =\overline{\mathcal{R}\left(A^{\Delta}\right)} X_{b} \oplus \overline{\mathcal{R}\left(A^{\Gamma}\right)} X_{b}^{\perp} \\
& =X_{b} \oplus X_{b}^{\perp} \quad\left(\text { since } \mathcal{R}\left(A^{\Delta}\right) \text { and } \mathcal{R}\left(A^{\Gamma}\right) \text { are dense in } X_{b} \text { and } X_{b}^{\perp}, \text { respectively }\right) \\
& =X .
\end{aligned}
$$

Therefore $\mathcal{R}(A)$ is dense in $X$. 
To show that $A$ is compact, we note that $A$ can be written in the form

$$
A=A^{\Delta} P_{X_{b}}+A^{\Gamma} P_{X_{b}^{\perp}}
$$

where $P_{X_{b}}$ and $P_{X_{b}^{\perp}}$ are the orthogonal projections of $X$ onto the subspaces $X_{b}$ and $X_{b}^{\perp}$, respectively. Since $A^{\Delta}$ and $A^{\Gamma}$ are both compact and $P_{X_{b}}$ and $P_{X_{b}^{\perp}}$ are both bounded, it follows that $A$, being the sum of two compact operators, is compact.

Consider now case II, that is, when $A$ is defined by (21). The operator $A$ is injective. In fact, if $A x=0$ then $A^{\Delta} x_{1}=-x_{2}$ and since $A^{\Delta} x_{1} \in X_{b}$ and $-x_{2} \in X_{b}^{\perp}$ we have that $A^{\Delta} x_{1}=-x_{2}=0$. Due to the fact that $A^{\Delta}$ is injective it follows that $x_{1}=0$, and therefore $x=x_{1}+x_{2}=0$.

Let us check now that $\mathcal{R}(A)$ is dense in $X$. By definition of $A$, and since $\mathcal{R}\left(A^{\Delta}\right) \subset X_{b}$, if follows that $\mathcal{R}\left(A^{\Delta}\right)$ and $X_{b}^{\perp}$ are subspaces of $X$ orthogonal to each other. Then

$$
\begin{aligned}
\overline{\mathcal{R}(A)} & =\overline{\mathcal{R}\left(A^{\Delta}\right)} \oplus \overline{X_{b}^{\perp}} \\
& =\left(X_{b} \cap \overline{\mathcal{R}\left(A^{\Delta}\right)}\right) \oplus X_{b}^{\perp} \quad\left(\text { since } \mathcal{R}\left(A^{\Delta}\right) \subset X_{b} \text { and } X_{b}^{\perp} \text { is closed }\right) \\
& =\overline{\mathcal{R}\left(A^{\Delta}\right)} X_{b} \oplus X_{b}^{\perp} \\
& =X_{b} \oplus X_{b}^{\perp} \quad\left(\text { since } \mathcal{R}\left(A^{\Delta}\right) \text { is dense in } X_{b}\right) \\
& =X .
\end{aligned}
$$

We will now show that also in this case the operator $A$ is compact. For that we write $A=A^{\Delta} P_{X_{b}}+P_{X_{b}^{\perp}}$. Since in this case $\Gamma$ is finite, if follows from (16) and (17) that $P_{X_{b}^{\perp}}$ is a finite rank operator and therefore, compact. Then, $A$ is compact since it is the sum of two compact operators.

Let us check now that in both cases (I and II) $b \in \mathcal{R}(A)$. Since $\hat{x} \in X_{b}$ and $A^{\Delta} \hat{x}=b$, it follows from (20) and (21) that $A \hat{x}=A^{\Delta} \hat{x}+A^{\Gamma} 0=b$ in case I, and $A \hat{x}=A^{\Delta} \hat{x}+0=b$ in case II.

Finally, let $x_{N}$ denote the least squares solution of $A x=b$ in $X_{N} \doteq$ $\operatorname{span}\left\{e_{1}, \ldots, e_{N}\right\}, x_{N}=\sum_{j=1}^{N} \xi_{j} e_{j}$. We will show that $\left\|x_{N}-\hat{x}\right\| \geq s(N)$ for every $N \in \mathbb{N}$.

$$
\begin{aligned}
\left\|x_{N}-\hat{x}\right\|^{2} & =\left\|\sum_{j=1}^{N} \xi_{j} e_{j}-\hat{x}\right\|^{2} \\
& =\left\|\sum_{j=1, j \in \Delta}^{N} \xi_{j} e_{j}+\sum_{j=1, j \in \Gamma}^{N} \xi_{j} e_{j}-\hat{x}\right\|^{2} \\
& =\left\|\sum_{j=1, j \in \Delta}^{N} \xi_{j} e_{j}-\hat{x}\right\|^{2}+\left\|\sum_{j=1, j \in \Gamma}^{N} \xi_{j} e_{j}\right\|^{2} \begin{array}{l}
\left(\text { since } \sum_{j=1, j \in \Delta}^{N} \xi_{j} e_{j}-\hat{x}\right. \\
\text { and } \left.\sum_{j=1, j \in \Gamma}^{N} \xi_{j} e_{j} \in X_{b}^{\perp}\right)
\end{array} \\
& \geq\left\|\sum_{j=1, j \in \Delta}^{N} \xi_{j} e_{j}-\hat{x}\right\|^{2} .
\end{aligned}
$$


For every $N \in \mathbb{N}$ define $k_{N} \doteq \max \left\{j: n_{j} \leq N\right\}$ or equivalently, $k_{N} \doteq \#\left\{e_{j}\right.$ : $\left.j \leq N, e_{j} \in B^{\Delta}\right\}=\#\left(B_{N} \cap B^{\Delta}\right)$, where $B_{N} \doteq\left\{e_{1}, \ldots, e_{N}\right\}$. It follows from (18) that $X_{k_{N}}^{\Delta}=\operatorname{span}\left\{e_{n_{j}}\right\}_{j=1}^{k_{N}}$. Since $X_{b}$ is a closed subspace of $X$ such that $X_{b}$ and $X_{b}^{\perp}$ are invariant under $A$ and $b \in X_{b}$, if follows from Lemma 3.5 that the least squares solution of $A x=b$ in $X_{b} \cap X_{N}=X_{k_{N}}^{\Delta}$ is

$$
P_{X_{b}} x_{N}=P_{X_{k_{N}}^{\Delta}} x_{N}=\sum_{j=1, j \in \Delta}^{N} \xi_{j} e_{j},
$$

where $x_{N}=\sum_{j=1}^{N} \xi_{j} e_{j}$ is the least squares solution of $A x=b$ in $X_{N}$.

Now, since $\left.A\right|_{X_{b}}=A^{\Delta}$ and $X_{k_{N}}^{\Delta} \subset X_{b}$, it follows that $A \eta=A^{\Delta} \eta \forall \eta \in X_{k_{N}}^{\Delta}$ and therefore $\sum_{\substack{j=\Delta \\ j \in \Delta}}^{N} \xi_{j} e_{j}$ is also the least squares solution of $A^{\Delta} x=b$ in $X_{k_{N}}^{\Delta}$, that is, $x_{k_{N}}^{\Delta}=\sum_{\substack{j=1 \\ j \in \Delta}}^{N} \xi_{j} e_{j}$ (see (18)). Then, substituting with this into (22) and using (19) it follows that

$$
\left\|x_{N}-\hat{x}\right\|^{2} \geq\left\|x_{k_{N}}^{\Delta}-\hat{x}\right\|^{2} \geq\left[\tilde{s}\left(k_{N}\right)\right]^{2}=\left[s\left(n_{k_{N}+1}\right)\right]^{2} .
$$

Now, by definition of $k_{N}$, if $j_{0} \in \mathbb{N}$ and $n_{j_{0}} \leq N$ then $k_{N} \geq j_{0}$. Therefore, the inequality $n_{k_{N}+1} \leq N$ would imply that $k_{N} \geq k_{N}+1$, which is a contradiction. Hence $n_{k_{N}+1}>N$ or, equivalently $n_{k_{N}+1} \geq N+1$.

Since $s$ is increasing and nonnegative it follows that

$$
\left[s\left(n_{k_{N}+1}\right)\right]^{2} \geq[s(N+1)]^{2} \geq[s(N)]^{2} .
$$

¿From (23) and (24) we finally conclude that $\left\|x_{N}-\hat{x}\right\| \geq s(N) \forall N \in \mathbb{N}$.

Next we show that the operator $A$ both in Theorem 3.4 and Corollary 3.6 can be chosen to further be self-adjoint.

Corollary 3.7. Let $X$ be an infinite-dimensional separable Hilbert space, $B \doteq\left\{e_{n}\right\}_{n=1}^{\infty}$ an orthonormal basis of $X, b \in X$ non-degenerate with respect to $B, X_{N} \doteq$ $\operatorname{span}\left\{e_{1}, \ldots, e_{N}\right\}$ and $s: \mathbb{N} \rightarrow \mathbb{R}^{+}$an arbitrary nonnegative increasing function. Then, there exists a compact, self-adjoint, injective, linear operator $A=A(b, s): X \rightarrow X$, whose range is dense in $X$ and a unitary operator $V: X \rightarrow X$ such that $b^{*} \doteq V b \in \mathcal{R}(A)$ and, if $x_{N}$ is the least squares solution of $A x=b^{*}$ in $X_{N}$, then $\left\|x_{N}-A^{-1} b^{*}\right\| \geq s(N)$ for every $N \in \mathbb{N}$.

Proof. Since $b \in X$ is non-degenerate with respect to $B$, it follows from Corollary 3.6 that there exists a compact, injective, linear operator $\tilde{A}=\tilde{A}(b, s)$ whose range is dense in $X$ such that $b \in \mathcal{R}(\tilde{A})$ and, if $x_{N}$ denotes the least squares solution of $\tilde{A} x=b$ in $X_{N}$, then $\left\|x_{N}-\tilde{A}^{-1} b\right\| \geq s(N)$ for every $N \in \mathbb{N}$. Let $\left\{\left(\sigma_{n} ; v_{n}, u_{n}\right)\right\}$ be the singular system associated to the compact operator $\tilde{A}$ and $V: X \rightarrow X$ the linear unitary operator defined by $V u_{n}=v_{n}$. Then, it can be easily proved that the linear operator $A \doteq V \tilde{A}$, besides being compact, injective and having range dense in $X$, is self-adjoint. This follows immediately from the fact that $V \tilde{A} v_{n}=\tilde{A}^{*} V^{*} v_{n}$ for all $n \in \mathbb{N}$. 
Since $b \in \mathcal{R}(\tilde{A})$, we have that $b^{*} \doteq V b \in \mathcal{R}(A)$. Now, since $\left\|A x-b^{*}\right\|=\|\tilde{A} x-b\|$, it follows that $x_{N}$ is also the least squares solution of $A x=b^{*}$ in $X_{N}$. Therefore, $s(N) \leq\left\|x_{N}-\tilde{A}^{-1} b\right\|=\left\|x_{N}-A^{-1} b^{*}\right\|$ for every $N \in \mathbb{N}$.

One may think that the result of Corollary 3.6 has little relevance from a practical point of view since it could happen that in a neighborhood of $b$, the only element $\eta \in \mathcal{R}(A)$ for which the least squares solutions in $X_{N}$ of $A x=\eta$ satisfying $\left\|x_{N}-A^{-1} \eta\right\| \geq s(N)$ for every $N \in \mathbb{N}$ is precisely $\eta=b$. However, this is not the case. In fact, it can be easily proved that in every reduced neighborhood of $b$ of radius $\delta$ there exists a sequence $\left\{b_{k}^{\delta}\right\}_{k=1}^{\infty} \subset \mathcal{R}(A)$ such that $b_{k}^{\delta} \rightarrow b$ as $k \rightarrow \infty$ and $\left\|x_{N}^{k}-A^{-1} b_{k}^{\delta}\right\| \geq s(N)$ for every $N \in \mathbb{N}$, where $x_{N}^{k}$ is the least squares solution of $A x=b_{k}^{\delta}$ in $X_{N}$. That is, for each element of the sequence $\left\{b_{k}^{\delta}\right\}_{k=1}^{\infty}$ (i.e. for each fixed $k)$, the approximating least squares solutions $\left\{x_{N}^{k}\right\}_{N=1}^{\infty}$, also diverges from the exact solution with speed arbitrarily large, in the same way that the least squares solutions obtained with data equal $b$ do.

Up to now we have only considered problems with exact data, that is, equations of the form $A x=b^{*}$, where $b^{*} \doteq A x^{*}$ and $x^{*}$ is the exact solution. As it is to be expected, the non-convergence in Corollary 3.6 can also occur with noisy data. Seidman ([1]) showed that if the operator $A: X \rightarrow X$ is compact (besides being linear, injective, positive, self-adjoint and with dense range), then for each increasing sequence of finitedimensional subspaces $\left\{X_{N}\right\}$ whose union is dense in $X$ and for each $b^{*} \in \mathcal{R}(A)$, there exists a sequence $b_{N} \rightarrow b^{*}$ for which the least squares solutions $x_{N}$ of $A x=b_{N}$ in $X_{N}$ satisfy $\left\|x_{N}-x^{*}\right\| \rightarrow \infty$, where $A x^{*}=b^{*}$. In a certain sense the next Corollary generalizes this result. More precisely, it is shown that also for the case of noisy data the careless application of the least squares method can lead to arbitrary rates of divergence.

Corollary 3.8. (Arbitrary divergence rate with noisy data) Let $X$ be an infinitedimensional separable Hilbert space, $B \doteq\left\{e_{n}\right\}_{n=1}^{\infty}$ an orthonormal basis of $X, b \in X$ non-degenerate with respect to $B, X_{N} \doteq \operatorname{span}\left\{e_{1}, \ldots, e_{N}\right\}, s: \mathbb{N} \rightarrow \mathbb{R}^{+}$an arbitrary nonnegative increasing function and $A=A(b, s)$ the operator whose existence was proved in Corollary 3.6. Then $\forall \alpha \in(0,1)$ there exists a sequence $\left\{b_{N}\right\}_{N=1}^{\infty} \subset \mathcal{R}(A)$ such that $b_{N} \rightarrow b$ as $N \rightarrow \infty$ and the least squares solution $x_{N}$ of $A x=b_{N}$ in $X_{N}$ satisfies $\left\|x_{N}-A^{-1} b\right\| \geq \alpha s(N)$ for every $N \in \mathbb{N}$.

Proof. Let $\left\{\alpha_{N}\right\} \subset \mathbb{R}$ be a sequence such that $\alpha_{N} \neq 0$ and

$$
\left|1-\alpha_{N}\right| \leq \min \left\{\frac{1}{\left\|x_{N}^{*}\right\|}, \frac{(1-\alpha) s(N)}{\left\|x_{N}^{*}\right\|}\right\}
$$

where $x_{N}^{*}$ is the least squares solution of $A x=b$ in $X_{N}$. By Corollary 3.6,

$$
\left\|x_{N}^{*}-A^{-1} b\right\| \geq s(N) \forall N \in \mathbb{N},
$$

then $\left\|x_{N}^{*}\right\| \rightarrow \infty$, which implies that $\left|1-\alpha_{N}\right| \rightarrow 0$ and therefore, $\alpha_{N} \rightarrow 1$ as $N \rightarrow \infty$. Let us define $b_{N} \doteq \alpha_{N} b$ for each $N \in \mathbb{N}$. Then, $b_{N} \rightarrow b$ and since $\mathcal{R}(A)$ is a subspace 
of $X,\left\{b_{N}\right\}_{N=1}^{\infty} \subset \mathcal{R}(A)$. Since the least squares solution $x_{N}$ of $A x=b_{N}=\alpha_{N} b$ in $X_{N}$ is $\alpha_{N} x_{N}^{*}$, it follows that

$$
\begin{aligned}
\left\|x_{N}-A^{-1} b\right\| & \geq\left\|x_{N}^{*}-A^{-1} b\right\|-\left\|x_{N}^{*}-x_{N}\right\| \\
& =\left\|x_{N}^{*}-A^{-1} b\right\|-\left|1-\alpha_{N}\right|\left\|x_{N}^{*}\right\| \\
& \geq s(N)-(1-\alpha) s(N) \quad(\text { from }(25) \text { and }(26)) \\
& =\alpha s(N) .
\end{aligned}
$$

Remark 3.9. During the proof above we made use of the following result: if $x_{N}$ is the least squares solution of $A x=b$ in $X_{N}$, then $\sigma x_{N}$ with $\sigma \neq 0$, is the least squares solution of $A x=\sigma b$ in $X_{N}$.

For simplicity we have only considered the case in which $X$ and $Y$ are real Hilbert spaces. However all results in this article carry over to the case of complex Hilbert spaces with the obvious modifications. Also, a procedure similar to the one employed in Corollary 3.6 can be used to show that the hypothesis of separability can be neglected.

\section{Conclusions}

In this article we have considered the application of the least squares method to inverse ill-posed problems of the form $A x=y$ where $A$ is a compact linear invertible operator on a separable Hilbert space $X$. It was shown that under no additional assumptions on the finite-dimensional subspaces there is no guarantee that the corresponding sequence of approximating least squares solutions will converge to the true solution. Moreover, it was shown that it is possible for the approximating solutions to diverge from the exact solution at any arbitrary speed, both for the case of exact and noisy data. In particular, Theorem 3.4 and Corollary 3.6 extend the original result of Seidman ([1], Example 3.1). The main difficulty that arises in these extensions resides in the construction, starting from the element $b \in X$ and the function $s(\cdot)$, of the sequences $\left\{\alpha_{n}\right\}_{n=1}^{\infty}$ and $\left\{\beta_{n}\right\}_{n=1}^{\infty}$ in such a way that they satisfy the constraints (4) and such that the operator $A$ defined from them by (5), satisfies $b \in \mathcal{R}(A)$ and $\left\|x_{N}-A^{-1} b\right\| \geq s(N)$ for all $N \in \mathbb{N}$.

The use of general residual minimization procedures, such as the least squares method, is very common in practice and it constitutes a widely used standard approach. Although the operators $A$ constructed in Theorem 3.4 and Corollary 3.6 are somewhat artificial they do serve to prove that in the case of ill-posed problems, the convergence of the finite-dimensional approximating solutions obtained by direct application of the least squares method is far from being automatically guaranteed, and that without any rigorous mathematical justification of this convergence, it is a completely unacceptable procedure. It is important emphasize here that the lack of convergence of the sequence of least squares solutions is not a consequence of the least squares method, but rather the result of a very poor choice of the approximating spaces $X_{N}$, without taking into 
account the operator $A$ which defines the ill-posed problem. Therefore, if the infinitedimensional problem is ill-posed, extreme care must be taken during discretization and finite-dimensional approximation of the solutions in order to guarantee convergence.

If the operator $A: X \rightarrow X$ is given, as it happens most of the times in practice, then Seidman [1] showed that for almost any $b \in \mathcal{R}(A)$ there exists a sequence of subspaces $X_{N}$ such that the corresponding sequence of least squares solutions of $A x=b,\left\{x_{N}\right\}$, is unbounded.

Although we strongly believe that the results in this article can also be extended to this case, i.e., that arbitrary rates of divergence can be obtained by the application of the least squares method when the operator $A$ is given, we are not able to provide a rigorous proof of this conjecture at this time. Efforts in this direction are underway.

\section{Acknowledgments}

This work was supported by Consejo Nacional de Investigaciones Científicas y Técnicas, CONICET, by Universidad Nacional del Litoral, U.N.L., and by Fundación Antorchas of Argentina. The authors also would like to thank an anonymous referee for many valuable comments and suggestions that have greatly improved the presentation of this article.

\section{References}

[1] T. I. Seidman, Nonconvergence Results for the Application of Least-Squares Estimation to Ill-Posed Problems, Journal of Optimization Theory and Applications, 30, 4, 535-547, 1980.

[2] H. W. Engl, M. Hanke and A. Neubauer, Regularization of Inverse Problems, Kluwer Academic Publishers, 1996.

[3] G. R. Luecke and K. R. Hickey, Convergence of approximate solutions of an operator equation, Houston Journal of Mathematics 11, 3, 345-354, 1985.

[4] C. W. Groetsch and A. Neubauer, Convergence of a general projection method for an operator equation of the first kind, Houston Journal of Mathematics 14, 2, 201-208, 1988.

[5] J. Weidmann, Linear Operators in Hilbert Spaces, Springer Verlag, 1980. 